\title{
Virtual Reality Applications and Language Learning
}

\author{
Panagiotis Panagiotidis \\ Aristotle University of Thessaloniki, Greece
}

\begin{abstract}
The development of Virtual Reality applications for educational use is not new, as this technology has attracted the interest of educators and scholars since its introduction to the market. However, in the last few years, technological advances in processing power, displays, graphics, mobiles and high-speed networks create new prospects for wider application of VR technology. This is why Extended (Virtual, Augmented and Mixed) Reality market, in the last few years is growing at a rapid pace. This also applies to the educational XR market which is also expected to experience a corresponding growth. Taking into account that the cost of equipment decreases, new opportunities for exploiting this technology in language education are appearing, and immersive technologies are becoming increasingly attractive to researchers and language experts.

This paper aims to present the current developments in the field of utilization of VR technology in language education and to explore its future perspectives. To this end, the theoretical bases of its use in language education, as well as the available hardware and software solutions are presented in more detail. Examples of VR applications, as well as the conclusions drawn from the literature review concerning the benefits and limitations of using VR systems in language learning are presented. Finally, future research directions are discussed, in order to identify the perspectives of Virtual Reality in language learning.
\end{abstract}

Keywords: Virtual Reality, Extended Reality, Immersive technologies, Virtual environments, Language Learning

\section{Introduction}

VR applications appeared in the late 60 s with I. Sutherland's development of the first head-mounted display [1]. Since then, VR has become very popular in many scientific fields (medicine, chemistry, biology, fluid dynamics etc), in industrial design (car and aircraft industry, architecture), the defense industry, the entertainment industry (movies, games) as well as in training and education. The term virtual reality was popularized by $\mathrm{J}$. Lanier, founder of the first company to sell VR products (VPL Research) and developer of sensor-based specialized equipment such as data suits and gloves and VR devices for medical, flight simulation, automobile industry design, and military training purposes. Carmigniani et al [1], define VR as a simulated 3D environment created by a computer, which completely immerses users in a synthetic world. VR technologies create a completely artificial environment and isolate the user from the outside world by reducing or eliminating the capacity to interact with the real world. This is achieved using a VR headset which allows users to see only the virtual environment and not the real world around them. VR technologies, also provide the user with the capacity to interact with the virtual environment. Lloyd et al [2], believe that VR "...replicates an environment and allows a simulation of the user to be present and interact in that environment"

VR is placed at one end of the "Virtuality continuum", as defined by Milgram and Kishino in 1994 [3], in which all applications described by the general term Extended Reality (XR) can be classified. This spectrum starts from computer generated virtual environments (VR) end ends in Augmented Reality (AR) applications, in which virtual content is superimposed in real-time atop (or above) physical objects in the real world. In that sense, VR is a more immersive experience, involving interactions with virtual objects within a completely synthetic environment [4].

In this paper, VR technology, the theoretical bases of its use in language education, as well as the available for its technology hardware and software solutions, are presented in more detail. Examples of VR technology applications, as well as the conclusions drawn from literature review concerning the benefits and limitations of using VR applications in language learning are also discussed.

\section{Theoretical background}

EDUCAUSE reports of the last two years, claim that Extended Reality technologies, can provide -a degree of- immersion to a simulated "authentic" environment, when applied in language learning, which is a particularly effective method for learning a language [5], [6]. Social interaction with native speakers, as well as practice and use of the target language in communicative situations, are essential components in language learning. However, this is not always possible, due to time, geographical, or 
financial restrictions. The ability of VR to overcome this kind of problems by providing users a simulated near-to-authentic environment is the main reason for its increasing popularity as a promising technology for language learning. Immersion allows language learners to combine learning a foreign language with an intercultural experience beyond geographical limitations with no need to visit the foreign country.

VR can create immersive environments, specifically developed to offer users contextual presence for language learning [7]. Immersion is essential in the language learning process Several commercial applications, such as Virtual worlds (Second Life), simulation games (The Sims) or massive multiplayer online games (WoW, LoL), and several custom VR applications have been used towards this direction. Literature of the analysis of findings in VR research published from 2004 to 2013 in the four top CALL journals (Language Learning \& Technology, CALICO Journal, Computer Assisted Language Learning, and ReCALL) conducted by Lin and Lan [7], revealed that the technology used most often in VR applications for language learning was open social virtualities $(65.6 \%)$, something actually expected, given the -earlier mentioned- ability of these environments to support interaction and communication between language users. The degree of interaction depends on the degree of immersion in the specific environment and the equipment in use. However, in VR the user's location is completely ignored. Depending on the equipment in use, his movement may also be ignored, or may be transposed to an avatar which represents the user in the virtual environment.

VR applications are based on several pedagogical theories such as ubiquitous learning, self-directed learning, constructivist learning, situated learning, inquiry-based learning, game-based learning, and engagement theory [8]. The technological innovation along with the flexibility it offers in pedagogical approach, are among the reasons why Virtual Reality (VR) is considered one of the most promising technologies for learning and training in the 21st century [9].

\section{Virtual Reality}

The most important notion -and target- in VR is the creation of a feeling of presence or "being there" in the virtual world [9]. This feeling is directly connected to the degree of immersion in the artificial environment. Immersion could be defined as the involvement in the environment, which causes lack of awareness of time and of the real world, as well as a sense of "being" in the task environment. However, different technologies are capable of creating different levels of immersion. In terms of immersive capabilities, a very common and early proposed classification is immersive versus non-immersive. A more accurate classification could distinguish three categories of VR systems. Non-immersive VR or desktop VR systems include a network connection and a desktop computer-based 3D graphical system, allowing the user to go through the VE using the mouse, the keyboard and the computer screen. This is usually the case of gaming software and the 3D virtual worlds. Li et al [10], use the term desktop VEs to refer to desktop-based virtual environments for gaming and education, to avoid confusion with the term desktop VR. These environments cannot create the sense of immersion as they are presented in two-dimensional (2D) computer screens. In those systems, immersion is usually mediated through the use of an avatar and the users have to imagine themselves in the virtual environment. In nonimmersive or desktop VR systems, users usually explore the environment from a third-person view via their avatars, although some non-immersive VR platforms offer a first-person or other points of view.

The second category, semi-immersive VR systems are more advanced installations with projectors and large screens, and -in some casesgesture recognition systems. In that case partial immersion may be achieved with the use of VR glasses or VR headsets to display the virtual environment. The degree of immersion varies, as it depends on the type of headset. Cave Automatic Virtual Environment (CAVE) systems are also considered to belong in this category, as the walls, the floor and ceiling, act as giant projection surfaces to create a highly immersive virtual environment [9].

Finally, fully immersive VR systems are headmounted systems where users' vision is fully covered, creating, thus, a sense of full immersion. Head Mounted Displays (HMDs) are headsets that visualize a natural realistic 3D space, without interference from the real world. HMDs project visual effects and multimedia directly to the eyes. Through the HMD, the user can see a spatial layout that is dynamically changing in accord with a firstperson's perspective (first-person view - FPV), which means that the view changes as the user moves his eyes or head as in real-life. Li et al [10], use the term iVR (immersive VR) and consider that at this stage "spatial presence" or "spatial immersion" is also achieved as the result of an increased level of immersion. iVR systems can support more realistic situations, such as interactions with the environment or with digital agents as well as user interactions with the appropriate equipment.

Interaction, is also an important aspect of VR. The effects of action performance in manipulating objects or navigating in virtual environments are important for student learning [10], [11]. Some VR systems, such as IMAX theaters which provide a high level of immersion through hi-end panoramic visualization, are not at all interactive as movies are passively received by the user. In terms of 
interaction, non-immersive systems, such as Desktop-based gaming VEs, allow some interactions even if these interactions need to be mediated through the avatar which represents the user. In that case, the limiting factor is the interface, as users participate via mouse and keyboard. This affects the activities which can be proposed in an environment such as Desktop VEs, including movement or performing actions (e.g. manipulating objects), as they must be controlled through this inconvenient for such a situation- interface. On the contrary, users can expect a high level of interaction in iVR systems. In "whole-body" simulations, interactions are almost realistic, as users participate through an HMD and have first-person view, wear equipment such as haptic gloves or motion sensors attached to their hands or feet, so they can manipulate objects using their hands, move using their own feet and perform actions enjoying a high level of immersion [10].

\subsection{Hardware and Software}

As mentioned above, to achieve the sensation of immersion in the artificial environment, VR systems usually use headsets or special peripherals. Consumer-oriented VR headsets emerged in the late 1980s, but cheaper and technically superior massmarket VR headsets, mostly developed for the gaming market, became widely available in 2016 . However, as there is a growing interest among scholars and researchers, most manufacturers also target the education market as well. VR Headsets can be experienced in stand-alone, computer-powered, or mobile-powered situations. Several devices available on the market could be classified into three categories, according to their possibilities and cost:

High-end market: The best available device in this category is Oculus Rift S (\$599) which totally immerses the user in an artificially generated world. In the same level are HTC VIVE (\$799) and HP Reverb G2 VR (\$599), as well as Oculus Quest 2 (\$299-\$399) and SONY Playstation VR (\$399). Headsets of this category dispose dedicated controllers as well as positional tracking systems, in order to track the user's movements into the physical space along the six axes of motion, making the VR experience more intuitive, "natural," and immersive. On the other hand, they require a tethered connection to a dedicated and personal computer (PC) which must be well equipped in order to deliver the expected hi-end result (estimated cost: $\$ 500$ graphics card plus $\$ 500-\$ 1,000$ worth of computing hardware). However, this combination can provide users with a more immersive, real-world-like type of experience through tracking user motion and simulating a virtual environment. Radianti et al [9], believe that the combination of some of these highend devices with data gloves or bodysuits (enhanced
VR applications) can deliver the highest level of immersion possible with consumer equipment.

Research on VR headsets is moving forward as major manufacturers are starting to release more affordable, untethered, positionally tracked VR devices with increasingly better quality. For example, the recently released Oculus Quest comes in the form of a cordless HMD, at the same price as the previous generation Rift with cables. New "standalone" low-cost devices are also becoming available on the market. These are hybrid AR/VR devices that do not require a PC or a mobile phone to operate, as they have integrated electronics, display, wifi and batteries. ClassVR is a complete VR and AR system for the classroom which comprises the equipment as well as a classroom management and control portal and the ability for schools to upload their own content $(\$ 250-400$ per headset + Teacher Portal Subscription / 12 months site license).

It must be noted that some of the equipment presented above, can potentially be useful in AR/MR applications. Manufacturers also tend to offer hybrid models that can cover VR and AR/MR applications too. The HTC VIVE, for example, is marketed as a VR headset, but some newer models contain forward-facing cameras that allow the user to view the physical world in the headset [5]. Radianti et al [9], in a recent literature review, found that $76 \%$ of the studies concerning the use of VR in language learning used HMDs of this category, such as Oculus Rift or HTC Vive while (20\%) used low-budget mobile VR headsets.

Mid-range market: in this category, all available devices are mobile powered. The best-known and advanced mid-range headset was Samsung's Gear VR (\$70) until Samsung discontinued the Samsung XR service and removed the Samsung VR Video app from Microsoft's and Oculus stores. The same is the case with Google Daydream View VR headset (\$99) which was also discontinued in 2019 and is no more supported by Google's new smartphones. Further choices are the Zeiss VR One (\$65) and the Homido device (\$30). Practically this market segment is vanishing, as the lower end devices offer almost the same functionality at a lower price.

Low-end market: At the lower end, there are smartphone-based VR solutions such as Google Cardboard viewers (\$10-\$20) and similar devices such as Homido Grab VR (€30) or Nintendo Labo ( $\$ 80$ VR Kit). These headsets work in combination with apps running on the smartphone which can display 3D scenes with stereoscopic rendering, track and react to head movements, and interact with apps by detecting when the user presses the viewer button. The VR content that can be viewed with Google Cardboard is usually a $360^{\circ}$ video or photo. In such an environment, users cannot walk to approach the objects around. The level of immersion is low, but it can be sufficiently engaging for educational use. 
Google Cardboard-type implementations are both promising and popular, as smartphones with sufficient computing power to render interactive educational 3D content stereoscopically are becoming cheaper.

Google Cardboard is directly associated with cheap and accessible tools like Google Expeditions [12], an immersive learning and teaching tool that many educators consider very useful in language education. Google Expeditions (2015) allows teachers to take an entire class on VR "field trips" or explore AR objects. Expeditions are $360^{\circ}$ photos annotated with descriptions and marked with specific points of interest called "spaces." Today, there are more than 900 tours available on the new platform 'Google Arts \& Culture'. Google Tour Creator (2018), a platform which allows users to create customized VR tours using Google Street View or their own $360^{\circ}$ photos taken with spherical cameras, is another popular VR app associated with Cardboard. The tours created can then be publicly published on Poly, Google's 3D content library, and viewed on mobile devices, desktop computers, or Cardboard. However, as 'Arts \& Culture' platform expands, Google plans to discontinue the Google Expeditions app as well as Tour Creator and the corresponding platform Google Poly, as of June 2021. Vortals, a new AR and VR design platform, is already promoted as the alternative solution.

A variety of web services or software applications and tools offer VR experiences. VR environments could be categorized based on their original design purposes into three types: open social virtualities or Virtual Worlds or MUVEs (Multiple User Virtual Environments), such as Second Life (SL), OpenSimulator, IMVU, and Active Worlds (AW), massive multiplayer online games (MMOGs) such as World of Warcraft, and Synthetic Immersive Environments (SIEs). Virtual Worlds allow the users to immerse themselves in a wide variety of social contexts and participate in individual or group activities, while MMOGs emphasize role-playing functions. In contrast, SIEs are especially designed for educational purposes and can be more suitable for this type of applications [7]. However, all VR environments provide opportunities for immersion in linguistic, cultural and task-based settings. While in Second life or OpenSim users can create their own virtual "islands" or worlds, there are also several VR creation tools that help users create their own VR objects or contexts / environments. Omni-Immersion Vision (OIV), Minecraft Realms, Tilt brush, Google Blocks, Thinglink, Cospaces are among the most known. Finally, a new generation of applications is beginning to arise enabled by new technological developments such as HTC Vivesync, a virtual meeting space, with direct access to One Drive and the possibility to integrate other business tools [10].

\subsection{Examples of VR applications}

VR has been attracting the attention of language educators and researchers for the last two decades as it offers obvious advantages in language learning. The most important is the possibility to create "authentic" contexts in which learners can immerse themselves by wearing 3D glasses or by using their avatars and interact with the objects in these contexts and with other learners [11]. These contexts can be $3 \mathrm{D}$ objects, scenes and simulations existing in the real world or fictional, or $360^{\circ}$ videos accessible via VR equipment. As a result, there is a wide range of applications with different approaches and aims. Lan [11], proposed a classification of VR applications for language education into five categories based on different pedagogical purposes: entertainment, social networking, visual experiences, creation, and operation. The examples presented below follow this classification.

The first category is dominated by VR games, the mainstream application of the VR industry. Immersive games, such as WoW, have been used as a medium for engaging learners in a real-time, ingame, and co-problem-solving context or provide them with out-of-school communities of practice in their target languages.

Concerning language learning, VR for social networking is the most promising category of applications, as it offers the opportunity for Reallife-like interaction. In the context of a virtual interdisciplinary approach, Arvanitis et al [13], proposed two scenarios (visits to a museum and a shopping center) for the development of oral and writing skills based on an OpenSim platform. Arvanitis and Panagiotidis [14], also proposed two communicative scenarios involving an accidental encounter in Second Life. In that case, the role of the native speaker of the foreign language is adopted by the instructor, who controls the communicative situation. Lan et al [15], used Second Life to create several virtual contexts, such as a zoo and two restaurants (western and Chinese), to help students of Chinese as a second language (CSL). Li et al [10], also used Second life to train American students on a set of Mandarin Chinese vocabulary. Xiao-Dong and Hong-Hui [16], in the framework of the VR-based blended flipped "English News" classroom, developed a distributed VR platform accessible through a cloud network to simulate a user's physical presence in a virtual or imaginary environment. Shih and Yang [17], created an immersive and interactive 3D virtual English classroom, entitled VEC3D, hosted in Activeworlds, to enhance learners' English communicative competence.

Applications based on Google Expeditions as well as on Discovery VR can be classified in the third category. In such applications, students can be involved in activities that go beyond the classroom 
and develop language skills mediated by technology. Chen et al [8], used Google Earth VR to develop writing skills of young learners in English. Parsons et al [12], used Cardboard VR and Google Expeditions (for VR) in combination with Actionbound (for outdoor location-based augmented reality), and Google Tour Builder (for augmented map-based learning) to create learning activities through which students can navigate using QR codes or GPS locations. Google Expeditions has developed over 100 VR trips for cultural visits, available to the public. Furthermore, the US Department of Education set out the EdSim Challenge, an initiative to promote the development of concepts for immersive simulations "that will spur an ecosystem of virtual and augmented reality technology in education" [10].

In the next category there are the applications that use VR Creation tools to help users create their own VR objects or contexts. Using tools such as OmniImmersion Vision (OIV) or Minecraft Realms, creators can participate in role-playing and interpersonal social interactions in the contexts they have created [11]. Minecraft is a very popular educational tool that supports text-based interpersonal interaction, as in addition to entertainment, it allows users to create their own virtual contexts and share them with others. Craft [18], successfully integrated Minecraft into his Latin classes engaging students in cooperative activities, Kervin [19], used Minecraft to observe literacy development of 16 children as they played and York [20], used Minecraft to develop Kotoba Miners $(\mathrm{KM})$, an environment for the acquisition of words or, more importantly, language, in particular Japanese and English.

The last category, VR for Operation, includes applications that offer manipulation of 3D objects and simulations of real-world processes. Simulations are very useful in language learning, as they offer the opportunity to involve users in educational scenarios. Howland et al [21], proposed Influent, a video game that uses a $3 \mathrm{D}$ environment to teach vocabulary words. Culbertson et al [22], developed Crystallize, an immersive 3D Japanese language learning video game where the player can collect words, use those words to converse with non-player characters, and collaborate with other players through avatars in joint tasks, or language "quests". Cheng et al [23], presented an adapted version of Crystallize which can be played in virtual reality with the Oculus Rift. The scope was to simulate the experience of being in a foreign language environment as deeply as possible, so that learners can learn both language and culture from observation. Results were positive, although problems such as motion sickness and dizziness were observed among users.

\section{Benefits for language learning}

VR technology, from an educational point of view, can offer a very important aspect of learning, that of personal experience and participation, when this is not practical or possible in the real world. To this end, as discussed in the previous paragraphs, a variety of VR applications have been developed for language education. Although in many of these studies, a specific pedagogical approach is not easily recognizable, there is an obvious sense that VR technology can offer several important benefits in language learning.

A common finding in several studies, is that VR applications can motivate students and lead to higher levels of interest and engagement in comparison to conventional teaching methods. Immersion seems to be an important factor in increasing students' motivation. In a systematic review of foreign language learning with immersive technologies, Hein et al [4], concluded that motivation and engagement were usually higher in the more immersive applications. They also noticed increased attention and enjoyment, as well as high acceptance of VR technology. Several other researchers [2], [8], also arrived at the conclusion that VR can be a useful addition to language education resulting in increased motivation and engagement.

The opportunities for authentic language tasks that VR can offer and the cultivation / improvement of learners' language and communicative skills as a result of their engagement in authentic, real-life learning scenarios, constitute a further category of benefits. Several researchers believe that VR can lead to enhanced learning [2], [8]. In their systematic review of studies concerning language learning with immersive technologies Hein et al [4], found that $50 \%$ of the studies used AR technologies, $24 \%$ were based on MUVE's, $13 \%$ on $360 \mathrm{o}$ videos, and $13 \%$ used fully immersive VR applications. Due to the fact that VR enables active participation and the exploration of distant and diverse learning contexts, and the feeling that the user is physically visiting and traveling to a place, the focus on VR based applications -as well as in MUVEs-, is on social interaction, communication, and intercultural learning experiences. In these applications the aim is not the promotion of specific language skills but the development of intercultural and language actionoriented communicative competence. Furthermore, Brown et al [6], believe that XR applications can provide learners with disabilities new kinds of access to education. Especially VR applications such as Virtual Worlds, can be particularly useful in assisting language learners with special learning needs, as they offer a low stress environment, potentially more suitable for students with difficulties in social interaction and communication, such as autism [7]. 
Several benefits concerning general learning skills related to language learning can also be noted. The promotion of interaction between participants (and/or the learning material, the teacher, or a virtual agent), is a common goal in VR applications, especially in communicative situations where opportunities for meaningful language practice arise. Li et al [10], reported enhanced behavioural and brain outcomes compared to traditional methods of classroom-based learning, Xiao-Dong and Hong-Hui [16], good teaching effects, and Mroz [24], language gains and increased critical thinking skills for the participants. Finally, Parmaxi [25], believes that VR can significantly increase students learning but also can help in the development of twenty-first century skills, although she also reports that there are cases where the use of VR has not brought any significant increase in student learning, possibly due to the complexity of 3D virtual learning environments.

\section{Discussion}

The technological innovation brought by XR technologies could lead to significant changes in education, comparable to those caused by the advent of PCs, the invention of web and the proliferation of mobile devices. Immersive technologies allow the real world to merge with the digital world enabling, thus, new forms of experience and the creation of engrossing simulated learning environments.

Each year VR systems are becoming more affordable and powerful, and their implementation can help reduce institutional costs, offering solutions to aspects where traditional - analog- education is costly. Training in laboratories or exchange programs, for example, could be substituted by virtual experiences via social VR applications, especially in unusual and unexpected social situations such as the pandemic [4]. Immersive learning environments can help in addressing the challenges of digitization in educational systems, assuming, of course, that schools are adequately equipped, and teachers have the digital skills and the positive attitude to adopt and use them creatively.

MarketWatch [26] reports that Global XR (VR, AR and MR) market's worth, was $\$ 25.4$ billion in 2019 , and with an increasing rate of $45.0 \%$ each year, is expected to reach $\$ 346.39$ billion by 2026 . Whilst innovative applications and high-speed wireless and cellular networks are expanding, the educational XR market is also expected to experience a corresponding growth. Wi-Fi 6 (802.11ax) and 5G, will make VR experiences more immersive and easily accessible to both local and remote learners.

Major technological companies such as Google, Facebook (with the acquisition of Oculus-Rift), Microsoft and Apple (New VR headsets / Glasses rumored for 2022) strengthen their position in the XP market with significant investments both in XR hardware and applications. These companies are primarily targeting gaming and entertainment, but the education sector also holds a very significant position in their plans. Therefore, there is a certainty that XR technologies will play an increasingly important role in education in the next few years and, consequently, in language education as well.

EDUCAUSE report for 2020 records a growing interest in exploring the potential of XR applications in teaching and learning. Language learning has a noticeable share in that market. Brown et al [6] reported that $2 \%$ of XR applications concern language learning. Radianti et al [9], had similar findings, performing a search for language learning VR applications in three main VR app stores, i.e., Steam, Vive, and Google Play. They finally evaluated 120 apps $3 \%$ of which was Language Learning apps. Statista [27] reports that the $66 \%$ of $\mathrm{XR}$ industry experts in the US, believe that immersive teaching experiences will be a major application of XR technologies in the education sector within the next two years followed by the applications aiming at the development of $21 \mathrm{st}$ century skills -collaboration, teamwork, problem solving- (57\%), interactive 3Dmodeling for learning $(55 \%)$, virtual travels in space and time (49\%), recreation /simulations (40\%), individualized learning $(32 \%)$ and facilitation of self-directed learning $(26 \%)$.

\section{Conclusion}

As equipment costs continue to decrease, immersive technologies are getting increasingly attractive to language experts. It is a fact that currently, VR systems are far from been widely adopted in classrooms. However, the future of VR technology in education, and particularly in language learning, seems very promising, as the cost of equipment decreases. In light of the growing research in VR technology, the efforts of language experts should focus on the pedagogical exploitation of this technology. An imaginative and innovative student-centered instructional design, offering collaborative interaction based on carefully designed educational scenarios, and activities that can place students in both formal and informal real-life situations, seems to be indispensable, in order to offer students useful learning environments that will help them develop their language skills.

\section{References}

[1] Carmigniani, J., Furht, B., Anisetti, M., Ceravolo, P., Damiani, E. and Ivkovic, M. (2011) 'Augmented reality technologies, systems and applications', Multimedia tools and applications, 51(1), pp. 341-377. 
[2] Lloyd, A., Rogerson, S. and Stead, G. (2017) 'Imagining the potential for using virtual reality technologies in language learning', In M. Carrier, R. M. Damerow, and K. M. Bailey (Eds.), Digital language learning and teaching: Research, theory, and practice, Routledge, New York, pp. 222-234.

[3] Milgram, P. and F. Kishino, F. (1994) 'A taxonomy of mixed reality visual displays', IEICE TRANSACTIONS on Information and Systems, 77(12), pp. 1321-1329.

[4] Hein, R. M., Wienrich, C. and Latoschik, M.E. (2021) 'A systematic review of foreign language learning with immersive technologies (2001-2020)', AIMS Electronics and Electrical Engineering, 5(2), pp.117-145.

[5] Pomerantz, J. (2019) 'XR for Teaching and Learning: Year 2 of the EDUCAUSE/HP Campus of the Future Project'. ECAR research report. EDUCAUSE, Louisville.

[6] Brown, M., McCormack, M., Reeves, J., Brook, D.C. Grajek, S., Alexander, B. and Weber, N. (2020) '2020 Educause Horizon Report Teaching and Learning Edition', EDUCAUSE, pp. 2-58.

[7] Lin, T.J. and Lan, Y.J. (2015) 'Language learning in virtual reality environments: Past, present, and future', Journal of Educational Technology and Society, 18(4), pp. 486-497.

[8] Chen, Y., Smith, T.J., York, C.S. and Mayall, H.J. 'Google Earth Virtual Reality and expository writing for young English learners from a Funds of Knowledge perspective', Computer Assisted Language Learning, 33:12, (2019), pp. 1-25.

[9] Radianti, J., Majchrzak, T.A., Fromm, J. and Wohlgenannt, I. (2020) 'A systematic review of immersive virtual reality applications for higher education: Design elements, lessons learned, and research agenda', Computers and Education, 147, 103778.

[10] Li, P., Legault, J., Klippel, A. and Zhao, J. (2020) 'Virtual reality for student learning: Understanding individual differences', Human Behaviour and Brain, 1(1), pp. 28-36.

[11] Lan, Y.J. (2020) 'Immersion, interaction, and experience-oriented learning: Bringing virtual reality into FL learning', Language Learning and Technology, 24(1), pp. 1-15.

[12] Parsons, D., Inkila, M. and Lynch, J. (2019) 'Navigating learning worlds: Using digital tools to learn in physical and virtual spaces'. Australasian Journal of Educational Technology, 35(4), pp.144-159.

[13] Arvanitis, P., Panagiotidis, P. and KigitsioglouVlachou, C. (2014) 'Interdisciplinarity in virtual worlds: teaching approaches for acquiring a language-culture', in Proceedings of 7th International Conference of Education, Research and Innovation, (ICERI 2014), IATED, Seville, Spain, pp. 3892-3901. ISBN: 978-84-617-2484-0, ISSN: 2340-1095.
[14] Arvanitis, and P. Panagiotidis, P. (2008) 'Language Learning in Virtual Worlds', in Proceedings of the International Congress '2008, European Year of Intercultural Dialogue', University Studio Press, Thessaloniki, pp. 97-108, ISBN 978-960-12-1910-3.

[15] Lan, Y. J., Lyu, B. N. and Chin, C.K. (2019) 'Does 3D immersive experience enhance Mandarin writing by CSL students?' Language Learning and Technology, 23(2), pp. 125-144.

[16] Xiao-Dong, L. and Hong-Hui, C. (2020) 'Research on VR-Supported Flipped Classroom Based on Blended Learning-A Case Study in-Learning English through New', International Journal of Information and Education Technology, 10(2), pp. 104-109.

[17] Shih, Y.C. and Yang, M.T. (2008) 'A collaborative virtual environment for situated language learning using VEC3D', Educational Technology and Society, 11(1), pp. 56-68.

[18] Craft, J. (2016) 'Rebuilding an empire with Minecraft: Bringing the classics into the digital space', The Classical Journal, 111(3), pp. 347-364.

[19] Kervin, L. (2016) 'Powerful and playful literacy learning with digital technologies', Australian Journal of Language and Literacy, 39(1), pp. 64-73.

[20] York, J. (2014) 'Minecraft and language learning', Minecraft in the Classroom: Ideas, inspiration, and student projects for teachers, pp. 179-196.

[21] Howland, R., Urano, S. and Hoshino, J. (2012) 'SanjigenJiten: computer assisted language learning system within a 3d game environment', Advances in Computer Entertainment, Springer, pp. 262-273.

[22] Culbertson, G., Andersen, E., White, W., Zhang, D. and Jung, M. (2016) 'Crystallize: An immersive, collaborative game for second language learning', in Proceedings of the 19th ACM Conference on ComputerSupported Cooperative Work and Social Computing, pp. 636-647.

[23] Cheng, A., Yang, L. and Andersen, E. (2017) 'Teaching language and culture with a virtual reality game', in Proceedings of the 2017 CHI Conference on Human Factors in Computing Systems, pp. 541-549.

[24] Mroz, A. (2015) 'The development of second language critical thinking in a virtual language learning environment: A process-oriented mixed-method study', Calico Journal, 32(3), pp. 528-553.

[25] Parmaxi, A. (2020) 'Virtual reality in language learning: A systematic review and implications for research and practice', Interactive Learning Environments, pp. 1-13.

[26] Marketwatch (2021). 'Extended Reality (XR) Market Size 2021 Industry Demand, Against Forecasting To 2030', Marketwatch; https://on.mktw.net/3ietZUg (28 May 2021). 
International Journal for Cross-Disciplinary Subjects in Education (IJCDSE), Volume 12, Issue 2, 2021

[27] Alsop, T. (2021) 'Top XR/AR/VR/MR applications in the education sector as per U.S. XR experts 2020'. Statista; https://bit.ly/3vxR20F (12 May 2021). 